\title{
RAABE
}

Die Autoren und Bücher des literarischen Expressionismus 


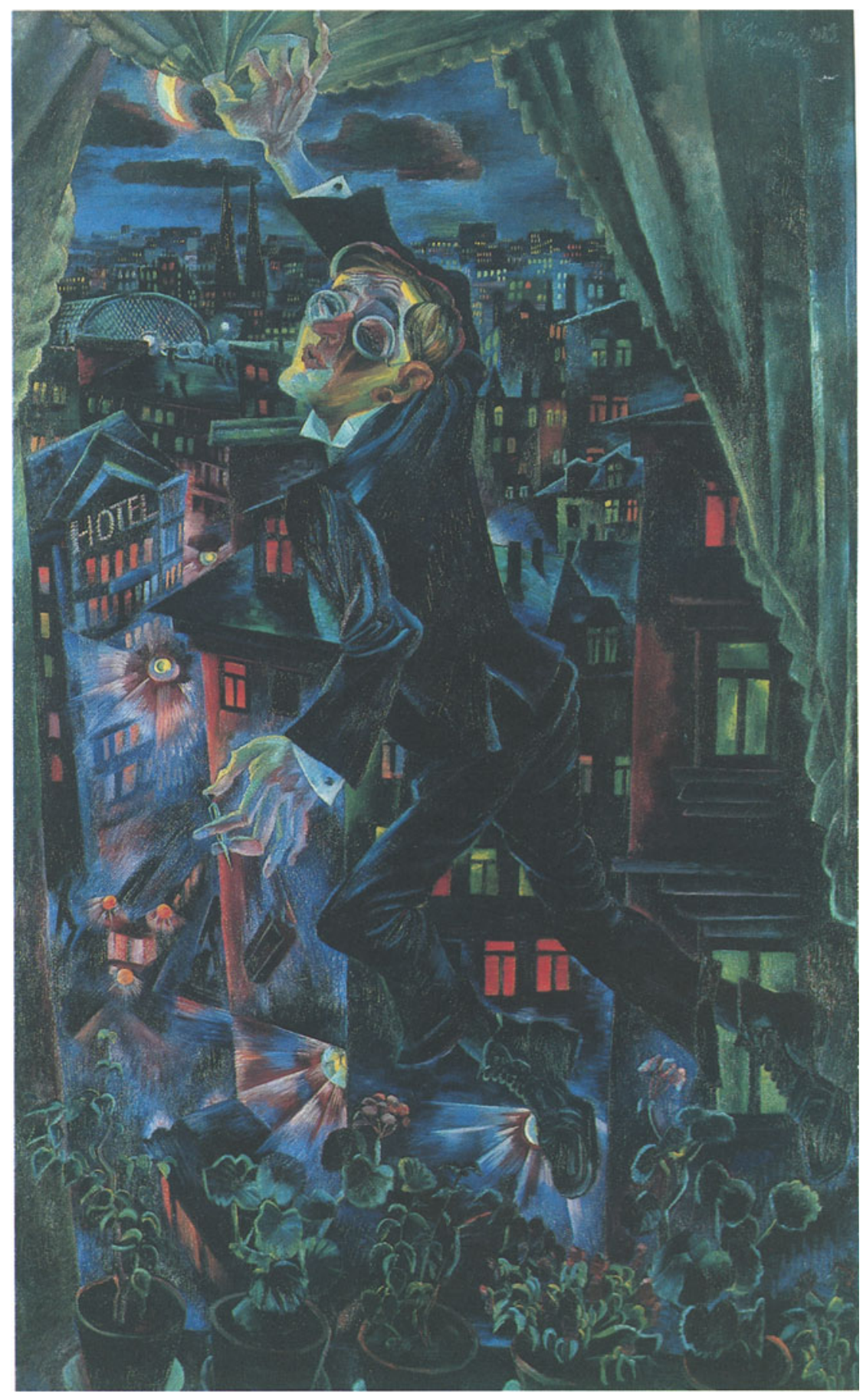




\section{Die Autoren und Bücher des literarischen Expressionismus}

Ein bibliographisches Handbuch in Zusammenarbeit

mit Ingrid Hannich-Bode

Zweite, verbesserte und um

Ergänzungen und Nachträge 1985-1990 erweiterte Auflage 
Die 1. Auflage wurde 1984, die 2. vorliegende Auflage am 1. Januar 1991 abgeschlossen.

\author{
Frontispiz: \\ Tod des Dichters Walter Rheiner. \\ Ölbild von Conrad Felixmüller. 1925. - \\ Mit freundlicher Genehmigung \\ von Robert Gore Rifkind \\ (Los Angeles/California).
}

Die Deutsche Bibliothek - CIP-Einheitsaufnahme

Raabe, Paul:

Die Autoren und Bücher

des literarischen Expressionismus :

ein bibliographisches Handbuch / Paul Raabe.

In Zusammenarbeit

mit Ingrid Hannich-Bode. -

2., verb. und mit Erg. und Nachtr. 1985-1990

erw. Aufl. - Stuttgart : Metzler, 1992

ISBN 978-3-476-00756-8

NE: HST

ISBN 978-3-476-00756-8

ISBN 978-3-476-03360-4 (eBook)

DOI 10.1007/978-3-476-03360-4

(C) 1992 Springer-Verlag GmbH Deutschland

Ursprünglich erschienen bei J.B. Metzlersche Verlagsbuchhandlung

und Carl Ernst Poeschel Verlag GmbH in Stuttgart 1992 
WILHELM BADENHOP

UND

KURT PINTHUS

zum Gedenken 


\section{Inhalt}

VORWORT IX

\section{EINLEITUNG}

Die Autoren und Bücher des literarischen Expressionismus 1

\section{A. LEXIKON}

Die Autoren und Bücher $A-Z$

\section{B. REPERTORIUM}

Die Autoren und Bücher des literarischen Expressionismus 561

\section{DIE AUTOREN}

1.1 Die Autoren nach ihren Geburtsdaten 563

1.2 Die Autoren nach ihren Geburtsorten 567

1.3 Berufsausbildung der Autoren 575

1.4 Fotos von Autoren 580

1.5 Promotionen 600

1.6 Weibliche Autoren 601

1.7 Lebensschicksale der Autoren 602

2 DIE BÜCHER 1910-1924

2.1 Chronologisches Repertorium 610

2.2 Alphabetisches Repertorium

2.3 Gattungsrepertorium 696

2.4 Verlagsverzeichnis 734

2.5 Illustratorenverzeichnis 815

2.6 Titelregister 835

\section{POSTUME VERÖFFENTLICHUNGEN}

3.1 Einzelveröffentlichungen (meist aus dem Nachlaß)

3.2 Teilsammlungen und Auszüge $\quad 872$

3.3 Teileditionen 874

3.4 Gesamtausgaben 875

\section{LEBENSZEUGNISSE}

4.1 Briefe und Briefeditionen

877

4.2 Tagebücher und Tagebuchaufzeichnungen $\quad 878$

4.3 Autobiographisches (Erinnerungen, Memoiren) 879

4.4 Weitere Dokumente 880

4.5 Nachlässe (nach Aufbewahrungsorten) 880

\section{C. ÜBERSICHTEN}

Die früheren und späteren Bücher der expressionistischen Autoren 883

1 DIE BÜCHER BIS 1910

1.1 Lyrik 885

1.2 Dramen 885

1.3 Erzählprosa 886

1.4 Sachprosa 887

1.5 Abhandlungen 887

1.6 Herausgegebene Schriften 888

1.7 Übersetzungen 888

1.8 Zeichnungen 888

2 DIE BÜCHER 1923/24-1932

2.1 Lyrik 889

2.2 Dramen 891

2.3 Erzählprosa 894

2.4 Sachprosa 899

2.5 Abhandlungen 903

2.6 Herausgegebene Schriften 904

2.7 Übersetzungen 906

2.8 Zeichnungen 909

2.9 Werkausgaben 909

3 DIE BÜCHER 1933-1945

3.1 Die im Ausland erschienenen Bücher 910

3.1.1 Lyrik 910

3.1.2 Dramen 911

3.1.3 Erzählprosa 911

3.1.4 Sachprosa 912

3.1.5 Abhandlungen 913 
3.1.6 Herausgegebene Schriften

3.1.7 Übersetzungen 914

3.1.8 Werkausgaben 914

3.2 Die in der Schweiz, Österreich und der Tschechoslowakei erschienenen Bücher 915

3.2.1 Lyrik 915

3.2.2 Dramen 915

3.2.3 Erzählprosa 915

3.2.4 Sachprosa 917

3.2.5 Abhandlungen 917

3.2.6 Herausgegebene Schriften

3.2.7 Übersetzungen 917

3.3 Die im Deutschen Reich erschienenen Bücher 918

3.3.1 Lyrik 918

3.3.2 Dramen 919

3.3.3 Erzählprosa 920

3.3.4 Sachprosa 923

3.3.5 Abhandlungen 924

3.3.6 Herausgegebene Schriften 926

3.3.7 Übersetzungen 926

3.3.8 Zeichnungen 927

\section{DIE BÜCHER NACH 1945}

4.1 Die im Ausland erschienenen Bücher 928

4.1.1 Lyrik 928

4.1.2 Dramen 929

4.1.3 Erzählprosa 929

4.1.4 Sachprosa 929

4.1.5 Abhandlungen 930

4.1.6 Herausgegebene Schriften

930

4.1.7 Übersetzungen 930

4.1.8 Werkausgaben 930

4.2 Die in der Schweiz und Österreich erschienenen Bücher 931

4.2.1 Lyrik 931

4.2.2 Dramen 931

4.2.3 Erzählprosa 932

4.2.4 Sachprosa 933

4.2.5 Abhandlungen 934

914
4.2.6 Herausgegebene Schriften

934

4.2.7 Übersetzungen 934

4.3 Die in Westdeutschland bzw. der Bundesrepublik und Westberlin erschienenen Bücher 935

4.3.1 Lyrik 935

4.3.2 Dramen 937

4.3.3 Erzählprosa 938

4.3.4 Sachprosa 942

4.3.5 Abhandlungen 945

4.3.6 Herausgegebene Schriften 946

4.3.7 Übersetzungen 949

4.4 Die in Ostdeutschland bzw. der

DDR erschienenen Bücher 951

4.4.1 Lyrik 951

4.4.2 Dramen 951

4.4.3 Erzählprosa 952

4.4.4 Sachprosa 952

4.4.5 Abhandlungen 953

4.4.6 Herausgegebene Schriften 954

4.4.7 Übersetzungen 954

\section{D.ERGÄNZUNGEN UND}

NACHTRÄGE 1985-1990

955

a. Lexikon. Die Autoren und Bücher A-Z 957

b. Repertorium 989 2 Die Bücher 1910-1924 989 3 Postume Veröffentlichungen 990 4 Lebenszeugnisse 992

c. Übersichten 994 1 Die Bücher bis 1910994

2 Die Bücher 1923/24-1932 994

3 Die Bücher 1933-1945 995

4 Die Bücher nach 1945996

E. ABBILDUNGSVERZEICHNIS UND BILDKONKORDANZ 999

F. ALLGEMEINES NAMENREGISTER 1009 


\section{Vorwort zur 1. Auflage}

Mit einer Verzögerung von vielen Jahren erscheint nunmehr, schon vor mehr als einem Jahrzehnt versprochen, dieses bibliographische Handbuch der Autoren und Bücher des literarischen Expressionismus. Angesichts dieser ungewöhnlichen Situation wird es erforderlich sein, Entstehung und Vorgeschichte der Bibliographie auch zum Verständnis ihrer Anlage und Zielsetzung vorauszuschicken. Ohne das beharrliche Drängen derjenigen, die an dem Werk von vornherein mitgewirkt haben und letzten Endes ohne das Gefühl der Verpflichtung den expressionistischen Autoren gegenüber, deren Namen, Lebensdaten und Werkverzeichnisse bewahrt werden sollten, wäre das Buch wohl nie erschienen. Bekanntlich ist die Vorbereitung einer Bibliographie, die sich ja auf Fakten beziehen muß, eine höchst undankbare und ihre Veröffentlichung eine höchst angreifbare Sache, die aber jeder kennt, der sich nicht scheut, dieses Geschäft allen Skrupeln und Zweifeln zum Trotz dennoch immer wieder einmal zu betreiben.

In der Einschätzung der bibliographischen Aufgabe weiß man sich mit vielen Wissenschaftlern und Autoren einig. Beschränken wir uns auf ein paar Zitate von Walter Benjamin, der nicht nur ein bedeutender Essayist war, sondern auch ein leidenschaftlicher Büchersammler und Bücherfreund. „Die Bibliographie", schreibt er, "ist eine Hilfswissenschaft. Und zwar steigt deren Wichtigkeit mit dem Steigen der Buchproduktion. Nun gibt es weniges, was für eine kritische Lage der Wissenschaft so durchaus charakteristisch ist wie der Umstand, daß dieser steigenden Wichtigkeit der Bibliographie ihre sinkende Beachtung seit Jahren parallel geht«. Was Benjamin vor fünfzig Jahren konstatierte, hat sich bis heute kaum zum Positiven verändert. Er fährt fort: "Die Bibliographie ist gewiß nicht der geistige Teil einer Wissenschaft. Jedoch sie spielt in ihrer Physiologie eine zentrale Rolle, ist nicht ihr Nervengeflecht, aber das System ihrer Gefäße. Mit Bibliogra- phie ist die Wissenschaft groß geworden, und eines Tages wird sich zeigen, daß sogar ihre heutige Krisis zum guten Teile bibliographischer Art ist «.

In der Tat hätte man angesichts der Möglichkeiten, die der bibliographischen Arbeit durch Informationssyssteme und Datenbanken, durch Großrechner und Mikrocomputer in der heutigen technischen Welt zur Verfügung stehen, erwartet, daß sich auch die deutsche Literaturwissenschaft dieser Chancen längst bedient haben würde, die gerade auch der Verzeichnung und Erschließung literarischer Texte aus Vergangenheit und Gegenwart zugute käme. Aber da es an bibliographischen Zentren für Geisteswissenschaften so gut wie ganz fehlt, ist der Einzelne nach wie vor gefordert, wobei er sich freilich die Chancen des Computers zunutze machen kann.

Bis heute gibt es eine bibliographische Sichtung und Erschließung der deutschen Literatur nach 1830, die sich an den hervorragenden Maßstäben des "Goedeke" messen könnte, nicht. Insbesondere fehlt es an Bibliographien zur modernen deutschen Literatur der letzten hundert Jahre. Abgesehen von einigen Forschungsfeldern, wobei man die Exilliteratur nach 1933 besonders hervorheben sollte, gibt es viele weiße Flecken auf der Landkarte der Literaturgeschichte.

Eine dieser Regionen ist der literarische Expressionismus, mein eigenes Forschungsgebiet seit drei Jahrzehnten, zu dem ich mit dem vorliegenden Buch nach langer Zeit zurückgekehrt bin. Die Wiederentdeckung dieser nach 1933 meist verfemten Autoren, die die literarische Szene in Deutschland am Vorabend des ersten Weltkrieges, in den Kriegsjahren und in dem Jahrfünft danach beherrschten, setzte bald nach dem zweiten Weltkrieg ein. Ich erinnere mich des starken Eindrucks, den Alfred Richard Meyers Buch "die maer von der musa expressionistica", Literaturgeschichte, Anthologie und Erinne- 
rungsbuch zugleich, auf mich machte, als es mir als Bibliotheksschüler im Sommer 1948 in Hamburg in die Hände fiel. Danach fand ich in Dr. Kurt Otte, dem Kubin-Sammler, einen unvergeßlichen Mentor, der mich auch mit den Hauptautoren des literarischen Expressionismus vertraut machte, die ich dann an der Hamburger Universität durch Hans Wolffheim und vor allem durch Karl Ludwig Schneider näher kennenlernte, dem ich an seiner Ernst Stadler-Ausgabe und dann bei der Herausgabe der Werke und Dokumente Georg Heyms einige Zeit half.

Als ich so vorbereitet 1958 Bibliothekar am Schiller-Nationalmuseum und dem gerade gegründeten Deutschen Literaturarchiv in Marbach wurde, erhielt ich im Schillerjahr 1959 den Auftrag, gemeinsam mit Ludwig Greve und Ingrid Grüninger eine Expressionismus-Ausstellung vorzubereiten, die, im Mai 1960 eröffnet, vor allem auch wegen ihres Katalogs großen Anklang fand. Wir lernten danach viele uns unbekannte Autoren und Bücher des Expressionismus kennen dank der Hilfe des Wuppertaler Sammlers Wilhelm Badenhop, dessen Expressionismus-Bibliothek Grundlage der Ausstellung wurde. Auch Hermann Kasack und Kurt Pinthus unterstützten unsere Arbeit, und nach der Ausstellungseröffnung lernten wir viele überlebende expressionistische Schriftsteller kennen, vor allem auch solche Autoren, die im Expressionismus-Katalog zu Unrecht nicht genannt worden waren.

Die Anregungen von vielen Seiten kamen den Sammlungen des Deutschen Literaturarchivs, aber auch den eigenen privaten Studien zugute. Die Zeitschriften des Expressionismus, verschollene Quellen zur Geschichte der literarisch-künstlerischen Bewegung, wurden $\mathrm{zu}$ einem Repertorium zusammengestellt, das in Buchform bei meinem Verleger Metzler in Stuttgart 1964 erschien. Der Cotta-Verlag hatte schon 1961 einen kommentierten Nachdruck der ersten vier Jahrgänge von Franz Pfemferts "Aktion « (1911-1914) ermöglicht: die vier weiteren Jahrgänge erschienen 1967 bei Kösel. Danach wurden von der Firma Kraus in Nendeln (Liechtenstein) sämtliche literarische Zeitschriften des Expressionismus nachgedruckt und schließlich 1972 durch ein 18-bändiges Repertorium, den »Index Expres- sionismus «, erschlossen, das mehr als 37000 Beiträge in fünf Reihen verzeichnet, eine Arbeit, die wir - meine Frau und ich - nur mit Hilfe der damals noch jungen nichtnumerischen Datenverarbeitung fertigstellen konnten.

Was aber noch ausstand, war die Sichtung der Autoren und die bibliographische Verzeichnung ihrer Buchveröffentlichungen. Auf der Grundlage des Expressionismus-Katalogs entstanden damals die ersten Listen, die ich auf meinen Reisen, auch ins Ausland - nach Amerika, Israel und Prag - in den sechziger Jahren ergänzte. Die Titel wurden vor allem an Hand der Sammlungen und Erwerbungen in Marbach vorläufig beschrieben. Dabei wurde ich seit 1966 vor allem durch eine meiner Marbacher Bibliothekarinnen, Ingrid Hannich-Bode, unterstützt. Sie fertigte die Titelaufnahmen nach einem gemeinsam erarbeiteten Schema an, vor allem ergänzte sie später die Bibliographie um die nach 1924/25 erschienenen Titel.

Infolge meines Abschieds aus Marbach 1968 und neuer Aufgaben in Wolfenbüttel geriet die Arbeit ins Stocken. Nur Ingrid HannichBode ließ sich nicht entmutigen, ergänzte und korrigierte die Titel. Erst vor drei Jahren wurde die Arbeit wieder intensiv aufgenommen und in einer letzten Phase das Werk zu Ende geführt, wobei ich den herzlichsten und aufrichtigsten Dank an Ingrid Hannich-Bode mit dem an meine Frau verbinden möchte, ohne deren tatkräftige Mithilfe und energische Unterstützung das bibliographische Unternehmen nicht hätte fertig werden können. Aus dem Dargestellten ergibt sich, daß die Bibliographie der Autoren und Bücher des literarischen Expressionismus aus den Quellen erarbeitet wurde. Die bekannten bibliographischen Hilfsmittel von Kosch bis WilpertGühring wurden benutzt und auch alle allgemeinen bibliographischen und biographischen Nachschlagewerke vom Deutschen Bücherverzeichnis bis zu Degeners "Wer ist's? " herangezogen. Die Bücher wurden in ihrem Hauptbestand aus der Phase des Expressionismus auf Grund von Autopsie beschrieben. Im übrigen wurden viele fehlende biographische Angaben in mühsamen Recherchen zusammengetragen, woran wiederum Ingrid Hannich-Bode einen Hauptanteil hat. 
Das vorliegende bibliographische Handbuch versucht, die an der literarisch- künstlerischen Bewegung des Expressionismus beteiligten Autoren zu bestimmen und ihre selbständig erschienenen Publikationen überschaubar zu machen. Das Werk beschränkt sich nicht auf ein alphabetisches Lexikon, sondern es wurde zugleich als Repertorium der Autoren und Bücher so angelegt, daß sich der Leser und Benutzer vom Umfang und Charakter der literarischen Überlieferung des Expressionismus und seiner Folgezeit bzw. seiner Vorgeschichte ein Bild machen kann. Die Bibliographie bezieht also auch die Büchertitel ein, die vor und nach dem Expressionismus erschienen sind. Das heißt also, daß ein Autor, der möglicherweise nur mit einer Buchveröffentlichung beteiligt war, dennoch mit der vollständigen Bibliographie seiner Publikationen in Buchform verzeichnet wurde. Auf diese Weise erfährt der Leser den Kontext, in dem die Buchproduktion und das literarische Leben des Expressionismus gesehen werden muß.

Ein Hauptproblem für den Bibliographen ist so auch im vorliegenden Fall - die Festlegung der Autoren, die in das Werk aufzunehmen waren. Angesichts der bekannten Schwierigkeiten in der Definition des Expressionismus kann es letzten Endes keine exakte, endgültige, sondern nur eine pragmatische Festlegung geben. Sie hängt in jedem Fall von dem Wissen und den Kenntnissen, auch von dem Epochenverständnis desjenigen $\mathrm{ab}$, der die Entscheidung zu treffen hat.

Die Bibliographie verzeichnet 347 Autoren, wesentlich mehr also, als man in einer Literaturgeschichte findet, und wesentlich weniger als die Namen aller Verfasser, die im "Index Expressionimus" vorkommen. Der Kern der verzeichneten Schriftsteller wird unstrittig sein: es sind die bekannten Hauptautoren, die jeder in einem Lexikon des Expressionismus erwartet. Auch die poetae minores, die Mitläufer und Mittöner, Randfiguren des literarischen Lebens, wird man akzeptieren, auch wenn sie vielleicht nur ein Buch veröffentlichten.

In anderen Fällen wird man streiten können: durfte man Franz Blei fortlassen, wenn man Erik-Ernst Schwabach einbezog? Konnte Wilhelm Lehmann ausgelassen werden, wenn man Fanz Kafka nennt? Diese Fragen wird man stellen, und sie verlangen eine klare Antwort. Entschieden wurde nach dem Verständnis der Zeitgenossen. Zum literarischen Expressionismus gehörig werden nicht nur die Expressionisten im engeren Sinne gesehen, also die Autoren, die im expressionistischen Stil schrieben, oder die, die Wortführer auf der einen und Randfiguren auf der anderen Seite waren, sondern alle Autoren, die sich mit dem literarischen Leben des Expressionismus identifizierten, sich daran beteiligten, deren $\mathrm{Na}$ men man in den Verlagsprogrammen und Schriftenreihen der Verlage findet, die sich für diese neue Literatur einsetzten und die man als Herausgeber und Mitarbeiter an Zeitschriften und Anthologien immer wieder gelesen hat. Im allgemeinen beschränkt sich das Lexikon auf Autoren, die mindestens ein Buch veröffentlichten oder herausgaben. Aber auch hier wurde nicht rigoros verfahren. Einige Autoren wie Rudolf Hartig, Hans Leybold, Hermann Plagge, Curt Saemann wurden dennoch einbezogen: zu einer Sammlung ihrer verstreut publizierten Arbeiten kam es weder damals noch später. Darüber hinaus aber lag es nicht in der Absicht dieses Werkes, jeden, der einmal ein paar expressionistische Gedichte in seinem Leben geschrieben hat, einzubeziehen.

Auf eine andere Schwierigkeit ist an dieser Stelle ebenfalls aufmerksam zu machen. Es hat sich eingebürgert, in der Periodisierung das "expressionistische Jahrzehnt « von 1910-1920 nicht exakt zu begrenzen, sondern die Ausläufer der expressionistischen Bewegung bis $1923 / 24 \mathrm{zu}$ akzeptieren. Auch in diesem Lexikon wurde grundsätzlich so verfahren. Auch hier verbot sich eine mechanische Zuweisung. Wenngleich der »Sturm « 1910 zu erscheinen begann, kann man kaum jedes Buch, das ein späterer Autor des Expressionismus in diesem Jahr veröffentlichte, ohne weiteres dem Neuen zurechnen. Auf der anderen Seite ist der Übergang vom Expressionismus zum Stil der zwanziger Jahre ebenfalls fließend, und so wurde auch in diesen Fällen, was nur für die Repertorien und Übersichten relevant ist, pragmatisch entschieden.

Schließen wir nach diesen grundsätzlichen Bemerkungen zum Kanon der Autoren und Bücher des literarischen Expressionismus 
einige Erläuterungen zur Anlage und zum Aufbau der Bibliographie an. Diese besteht aus drei Teilen: dem Lexikon, dem Repertorium und den Übersichten. Darüberhinaus werden die Bebilderung und das Gesamtregister als wesentliche Elemente des Buches angesehen.

Der Teil A - das Lexikon der Autoren und Bücher des literarischen Expressionismus - verzeichnet die Autoren in alphabetischer Reihenfolge nach ihren gebräuchlichen Namen, wobei von Pseudonymen verwiesen wird. Jeder Artikel besteht aus den Lebensdaten des Autors, der Bibliographie seiner selbständig erschienenen Bücher, die er verfaßt, herausgegeben, eingeleitet oder übersetzt hat, und den bibliographischen Hinweisen auf Nachlaß und Sekundärliteratur.

Die Lebensdaten beschränken sich bewußt auf wenige biographische Angaben. Es war nicht beabsichtigt, die Autoren in ihrer individuellen Bedeutung $\mathrm{zu}$ charakterisieren, zumal solche Interpretationen subjektiv ausfallen müßten. Die Aufnahme in das Lexikon bezeichnet eine Zuweisung zur Literatur des Expressionismus. Es bleibt dem Benutzer und Leser überlassen, sich an Hand der Werke des Autors ein Bild seines Schaffens zu machen. Viele biographische Angaben wurden neu ermittelt. Dennoch konnten bisher nicht alle Lebensdaten trotz großer Bemühungen festgestellt werden. Es ist zu hoffen, daß diese Veröffentlichung zu weiteren Nachforschungen anregt.

Den Lebensdaten folgt die Bibliographie der Buchveröffentlichungen des Autors in chronologischer Anordnung. Die Einzelausgaben aus der Phase des Expressionismus werden ausführlicher unter Angabe bibliographischer Einzelheiten beschrieben, als dies bei den übrigen Büchern der Fall ist. Die von einem Autor herausgegebenen oder übersetzten $\mathrm{Pu}-$ blikationen schließen sich dem Verzeichnis der Einzelausgaben an. Danach werden ggf. Werkausgaben, Brief- und Tagebuch-Editionen aufgeführt.

Am Ende eines Artikels findet man ggf. einige zusätzliche Anoaben: den Hinweis auf den Nachlaß des Autors und auf einen oder ein paar Titel der Sekundärliteratur. Die Verzeichnung der Literatur über den Autor wurde nicht als Aufgabe dieser Bibliographie betrachtet. Wünschenswert und notwendig wäre eine
Bibliographie der Literatur über den literarischen Expressionismus im allgemeinen und über die einzelnen Autoren im besonderen: sie könnte dieses Werk aktuell ergänzen.

Der Teil B - das Repertorium der Autoren und Bücher des literarischen Expressionismus - hat die Aufgabe, die biographischen und bibliographischen Angaben des Lexikons, sofern sie sich auf die Zeit des Expressionismus beziehen, aufzuschlüsseln und überschaubar zu machen. Das Repertorium ist der Versuch einer statistischen Sichtung biographischer und bibliographischer Fakten.

Im ersten Abschnitt werden die biographischen Angaben zu den einzelnen Autoren in sechs Übersichten aufgelistet. Die Autoren werden nach ihren Geburtsdaten, Geburtsorten, Ausbildungswegen und Lebensschicksalen verzeichnet. Die lange Liste der Promotionen kam hinzu und die auffallend knappe Übersicht über die schreibenden Frauen, die an der Literatur des Expressionismus beteiligt waren.

Im zweiten Abschnitt des Repertoriums werden die Bücher des literarischen Expressionismus nach verschiedenen Gesichtspunkten in Kurzform aufgelistet. Man kann sich so einen Überblick über die Buchproduktion machen und sich den Stellenwert eines Autors, die Bedeutung bestimmter Erscheinungsjahre, Gattungsformen und Verlage vergegenwärtigen. Unter Zugrundelegung eines sehr einfachen Gattungsschemas - Lyrik, Dramen, Erzählprosa, Sachprosa, Wissenschaftliche Arbeiten, Herausgegebene Schriften, Übersetzungen, Werkausgaben, Zeichnungen und Graphik - wurden die Titel zu einem chronologischen, einem alphabetischen und speziellen Gattungsrepertorium zusammengestellt. Es folgt ein Verlagsverzeichnis mit Aufführung der Buchveröffentlichungen aus der Zeit des Expressionismus, wobei die Gründungsdaten, Inhaber etc., ggf. Literaturangaben den einzelnen Firmen, soweit ermittelt, beigefügt sind. Die illustrierten Bücher des Expressionismus werden in einem weiteren Verzeichnis mitgeteilt. Ein Titelregister schließt den Abschnitt ab.

Das sechsfache Repertorium wurde mit Hilfe eines Mikrocomputers vom Typ Sirius ermöglicht, wofür ich meinem Kollegen Dr. Erdmann Weyrauch und seinem Sohn Sebastian 
herzlich danke. Sie haben keinen Aufwand gescheut, ein komplettes Programm für dieses Werk auszutesten. Mit Hilfe des Computers konnten so Übersichten gedruckt werden, zu denen sich der Bibliograph mit konventioneller Arbeitstechnik kaum entschlossen hätte. Die Bücher des literarischen Expressionismus werden auf diesem Wege in ihrer Gesamtheit $\mathrm{zu}$ einer faßbaren und vergleichbaren Größe, die sich statistisch auswerten läßt und Vermutungen und Annahmen exakt untermauern kann.

Der technische Aufwand, der für die Mehrfachverzeichnung sinnvoll war, war bei den beiden folgenden Abschnitten des Repertoriums nicht notwendig, in denen die postumen Veröffentlichungen expressionistischer Werke in Einzelausgaben und Gesamtausgaben verzeichnet wurden. Der Stand der Editionen expressionistischer Texte sollte hier dokumentiert werden. Einem orientierenden Zweck dient auch die Zusammenstellung der Lebenszeugnisse im vierten Abschnitt: das Verzeichnis der Briefe und Briefeditionen, der Tagebücher, Autobiographien und Dokumente ergibt eine Quellenübersicht, die durch die Nennung der Aufbewahrungsorte von Nachlässen abgerundet werden sollte.

Der Teil C - die Übersichten der früheren und späteren Bücher der expressionistischen Autoren sichtet die Werke der Schriftsteller vor 1910 und nach 1923/24. Wieder wurde ein einfaches Gattungsschema zugrundegelegt, die Titel aber nur jeweils in den einzelnen Zeitabschnitten - vor 1910, 1923/24-1932, 1933-1945, nach 1945 - chronologisch verzeichnet. Für das Verständnis der expressionistischen Autoren und auch zum Verständnis der expressionistischen Bewegung selbst mag es hilfreich sein, sich die Buchproduktion vor und nach dem Expressionismus zu vergegenwärtigen, sofern die Autoren dieser Zeit selbst daran beteiligt waren. Man erfährt auch einiges über das Schicksal der Verfasser, wenn man sieht, wie sich nach 1933 die Wege trennten.

Die Absicht dieses bibliographischen Handbuches besteht in einer möglichst vielschichtigen Information des Lesers und Benutzers, die über die übliche bibliographische Verzeichnung hinausgeht und so einer literaturgeschichtlichen Darstellung des Expressionismus neue Daten und Fakten an die Hand geben kann.

Man wird, wenn man sich mit den in diesem Lexikon enthaltenen Nachweisen näher beschäftigt, Grundlagen für eine Geschichte der expressionistischen Buchkultur finden. Wenngleich die Buchveröffentlichungen, um die es hier geht, meist nicht den optischen Reiz der Zeitschriften des Expressionismus erreichen, lernt man doch aus den Titelblättern und Illustrationen den Geist der Zeit kennen, beispielsweise das Vorherrschen der Antiqua und des Holzschnitts. Aus diesem Grunde wurde vor allem der lexikalische Teil des Werkes mit Reproduktionen von Titelblättern und Illustrationen ausgestattet, darüber hinaus wurden beispielhaft und in Auswahl einige graphische Porträts expressionistischer Autoren, gezeichnet, gestochen oder in $\mathrm{Holz}$ geschnitten von expressionistischen Künstlern, abgebildet. So mögen die geistigen und persönlichen Beziehungen zwischen Dichtern und Malern, Literaten und Graphikern aufscheinen. Auch kann so etwas wie ein "SoergelEffekt « erreicht werden: durch das Abbildungsmaterial in Albert Soergels Literaturgeschichte "Im Banne des Expressionismus" (1925) ist ja diese Periode deutscher Literatur über die Zeiten hinweg lebendig geblieben. Deshalb wurde neben der Wiedergabe einiger Dokumente eine Serie von Photos in das Repertorium eingefügt: wiederum an Beispielen sollen die Gesichter dieser expressionistischen Generation ein wenig die Welt widerspiegeln, die sich dem Leser erst eröffnet, wenn er sich mit den Texten beschäftigt, zu deren Lektüre dieses Lexikon letztlich hinführen möchte.

Das bibliographische Handbuch der Autoren und Bücher des literarischen Expressionismus ist der Versuch, die Bedeutung dieser großen kulturellen Bewegung am Beginn des 20. Jahrhunderts durch Fakten und Titel, Bilder und Übersichten anzuzeigen. Es geht nicht um eine Darstellung, sondern um Informationen, bibliographische Hinweise, Sicherung von Lebensspuren als Voraussetzung für die Darstellung geschichtlicher Zusammenhänge. Sicherlich wird dieses Handbuch nicht allen Wünschen der Kenner und Bücherfreunde, der Leser und Benutzer gerecht werden 
können. Aber es war der Ehrgeiz des Bibliographen, einen neuen Zugang zur Bücherwelt des Expressionismus und zur Lebenswelt ihrer Autoren zu eröffnen. Nach der Erschließung der Zeitschriften und Sammlungen ist es an der Zeit, die Hauptwerke des Expressionismus, die Bücher, zu beschreiben, ihre Titel zusammenzustellen und so die Zeit des literarischen Expressionismus insgesamt überschaubar zu machen.

Dieses Werk kann so die ExpressionismusForschung zu weiteren Untersuchungen und Überlegungen anregen, vor allem aber soll es dazu beitragen, dieser Epoche neue Freunde zuzuführen und die Erinnerung an diese immer noch aufregende und anregende Phase der Literatur- und Kulturgeschichte lebendig zu erhalten.

Am Ende sei der Dank an Ingrid HannichBode und Mechthild Raabe, meine Frau, wiederholt: ihnen vor allem ist es, wie schon gesagt, zuzuschreiben, daß dieses Buch endlich erscheinen kann. Wir danken allen Institutionen, Bibliotheken, Sammlungen, Archiven, die uns Auskünfte gegeben haben, allen Personen, die unsere Nachforschungen unterstützten, namentlich auch Wulf Kirsten (Weimar) und Peter Ludewig (Berlin/DDR). Ohne die stets freundschaftliche Hilfe aller
Mitarbeiter des Deutschen Literaturarchivs in Marbach wäre dieses Werk nicht vorangekommen: Bernhard Zeller, Ludwig Greve, Reinhard Tgahrt und Werner Volke danken wir stellvertretend für viele. Ein Wort des Dankes gilt schließlich Robert Gore Rifkind (Los Angeles, Californien), dessen hervorragende Expressionismus-Sammlung mir in der Endphase der Arbeit offenstand.

Dem Metzler Verlag, mit dem ich seit 25 Jahren zusammenarbeite, gilt mein aufrichtiger Dank für die Mühe und Geduld bei der Herstellung dieses Werkes, namentlich den Lektoren Bernd Lutz und Uwe Schweikert, und schließlich dem Hersteller Axel Eberhard. Das Werk widme ich zwei großen verstorbenen Expressionismusfreunden, die mir in meinem Leben und im Umgang mit den Werken des literarischen Expressionismus viel bedeutet haben und derer ich in Dankbarkeit, Freundschaft und Verehrung gedenke: Wilhelm Badenhop (1902-1961), dem ersten großen Sammler expressionistischer Bücher, und Kurt Pinthus (1886-1975), dem Herausgeber der "Menschheitsdämmerung" und lebenslangen Verkünder der Taten des Expressionismus.

Wolfenbüttel, im Februar 1985 Paul Raabe 


\section{Vorwort zur 2. Auflage}

Die vorliegende zweite Auflage dieses bio-bibliographischen Handbuchs der Autoren und Bücher des literarischen Expressionismus, das von der Kritik freundlich aufgenommen wurde, beschränkt sich auf Korrekturen und Ergänzungen sowie auf Nachträge für die Zeit von 1985 bis 1990. Da der Umbruch des Werkes gegenüber der ersten Auflage von 1985 aus technischen und finanziellen Gründen nicht verändert werden konnte, ließen sich im Hauptteil lediglich Korrekturen und einige wenige Ergänzungen und Verbesserungen einbringen. Das gilt sowohl für das Lexikon wie für das Repertorium und die Übersichten. Alle größeren Ergänzungen, Berichtigungen und Nachträge für die Zeit von 1985 bis 1990 wurden im Teil D des Werkes zusammengefaßt. Der Lexikonteil (A) wird so mit diesem Anhang (D) verknüpft, daß der Benutzer zu allen mit einem Sternchen $\left(^{*}\right)$ versehenen Autorennamen Ergänzungen und Nachträge auf den Seiten 957-988 finden kann. Es empfiehlt sich also, Hauptteil und Anhang gleichermaßen zu benutzen. Nachträge zu dem Repertorium (B) und den Übersichten (C) sind auf S. 989-997 verzeichnet.

Der Verfasser konnte sich nicht entschließen, den Kanon der Autoren des Expressionismus wesentlich zu erweitern. So wurden nach sorgsamer Abwägung nur sechs Namen hinzugefügt: Lili von Braunbehrens, Paul Duysen, Joseph Gregor, John Höxter, Wassily Kandinsky und Leopold Wolfgang Rochowanski. (Die drei Sternchen verweisen im Lexikonteil auf diese Namen im Anhang.) Da ich beabsichtige, ein vor 25 Jahren erschienenes Werk über die Zeitschriften und Sammlungen des literarischen Expressionismus neu zu bearbeiten und um die Kunstzeitschriften und illustrierten Bücher des Expressionismus zu erweitern, wird dies eine Gelegenheit sein, vor allem die Herausgeber und Künstler, die im $\mathrm{Zu}$ sammenhang mit dem Expressionismus stan- den, auch biographisch und bibliographisch nachzuweisen.

$\mathrm{Zu}$ Mißverständnissen können die Literaturhinweise Anlaß geben. Bewußt habe ich mich auf solche Titel beschränkt, die das bibliographische Rüstzeug ergänzen. Eine Bibliographie der Forschungsliteratur zu den Autoren des Expressionismus bleibt leider weiterhin ein Desideratum.

Es versteht sich, daß das Namensregister selbstverständlich alle im Hauptteil wie in den Nachträgen vorkommenden Namen umfaßt. Die Benutzung des Werkes wird durch dieses Gesamtregister erleichtert.

Für Hinweise und Nachträge habe ich vielen aufmerksamen Lesern, Rezensenten, Antiquaren, Sammlern und Bibliothekaren zu danken. Namentlich nenne ich Jörg Drews (Bielefeld), Walter Fähnders (Osnabrück), Gerd W. Jungblut (Schlitz), Wulf Kirsten (Weimar), IngoWolf Kittel (Heidelberg), Michael Matzigkeit (Düsseldorf), Volker Pirsich (Hamburg), Karl Riha (Siegen), Lutz Schulenburg (Hamburg), Werner J. Schweiger (Wien), Jürgen Serke (Hamburg), Gerrit Walther (Frankfurt a. M.), Gerhard Wietek (Hamburg) und Carl Winter (Heidelberg). Auch haben mich viele Verleger, bei denen Rückfragen notwendig waren, freundlich unterstützt und mir teilweise sogar die fraglichen Werke überlassen. Auch hierfür möchte ich herzlich danken. Frau Dr. Ingrid Hannich-Bode (Basel) hat für die Neuauflage die von ihr gesammelten Ergänzungen beigetragen. So gilt auch ihr mein herzlicher Dank. Es freut mich, daß der Metzler Verlag den Wunsch geäußert hat, diese Neuauflage herauszugeben. Ich habe ihn gern erfüllt in der Hoffnung, daß das Werk auch in dieser verbesserten und ergänzten Ausgabe die Expressionismusforschung fördern wird.

Wolfenbüttel, im Februar 1991 P.R. 